PROCEEDINGS OF THE

AMERICAN MATHEMATICAL SOCIETY

Volume 133, Number 6, Pages 1699-1706

S 0002-9939(04)07747-0

Article electronically published on December 20, 2004

\title{
GRADIENT RANGES OF BUMPS ON THE PLANE
}

\author{
JAN KOLÁŘ AND JAN KRISTENSEN
}

(Communicated by David Preiss)

\begin{abstract}
For a $\mathcal{C}^{1}$-smooth bump function $b: \mathbb{R}^{2} \rightarrow \mathbb{R}$ we show that the gradient range $\nabla b\left(\mathbb{R}^{2}\right)$ is the closure of its interior, provided that $\nabla b$ admits a modulus of continuity $\omega=\omega(t)$ satisfying $\omega(t) / \sqrt{t} \rightarrow 0$ as $t \searrow 0$. The result is a consequence of a more general result about gradient ranges of bump functions $b: \mathbb{R}^{n} \rightarrow \mathbb{R}$ of the same degree of smoothness. For such bump functions we show that for open sets $G \subset \mathbb{R}^{n}$, either the intersection $\nabla b\left(\mathbb{R}^{n}\right) \cap G$ is empty or its topological dimension is at least two. The proof relies on a new Morse-Sard type result where the smoothness hypothesis is independent of the dimension $n$ of the space.
\end{abstract}

\section{INTRODUCTION}

A real-valued $\mathcal{C}^{1}$ function $b: \mathbb{R}^{n} \rightarrow \mathbb{R}$ is called a bump if its support, $\operatorname{spt}(b)$, defined as the closure of the set $\left\{x \in \mathbb{R}^{n}: b(x) \neq 0\right\}$, is bounded and non-empty. This work is part of an attempt to find characteristic properties, topological and otherwise, of the gradient range $\nabla b\left(\mathbb{R}^{n}\right)$ of a $\mathcal{C}^{1}$-smooth bump $b$ on $\mathbb{R}^{n}$. Only the case $n \geq 2$ is interesting since for $n=1$ it is easy to see that the gradient ranges are precisely those bounded and closed intervals that contain $0 \in \mathbb{R}$ in their interior. Here we address the particular question of whether the gradient range of a $\mathcal{C}^{1}$-smooth bump $b: \mathbb{R}^{n} \rightarrow \mathbb{R}$ is the closure of its interior (i.e., is regularly closed). Previous works related to this question include [1, 2], 3], 5] and 6]. In particular, we emphasize that for $\mathcal{C}^{2}$-smooth bumps $b: \mathbb{R}^{n} \rightarrow \mathbb{R}$ (in any finite dimension $n$ ) one can use Th. 1, Sect. 3 of [5] in connection with a straightforward argument based on the Inverse Function Theorem to show that $\nabla b\left(\mathbb{R}^{n}\right)$ is regularly closed. It is however not clear how to relax the $\mathcal{C}^{2}$-smoothness condition imposed in [5]. In the infinite-dimensional case the gradient range is far from being regularly closed. Indeed, there exists a $\mathcal{C}^{1}$-smooth bump $b: \ell_{2} \rightarrow \mathbb{R}$ such that $\nabla b\left(\ell_{2}\right)$ has empty interior (see [1]). In [6] it is shown that the gradient range of a $\mathcal{C}^{1}$-smooth bump $b$ on the plane is regularly closed and has a connected interior provided that either

(i) $\nabla b$ is of bounded variation, or

(ii) $\nabla b$ admits a modulus of continuity $\omega=\omega(t)$ satisfying $\int_{0}^{1} \frac{d t}{\omega(t)}=\infty$.

The corresponding $n$-dimensional results are weaker: the gradient range of a $\mathcal{C}^{1}$ smooth bump on $\mathbb{R}^{n}$ satisfying either (i) or (ii) is at least (topologically) twodimensional at all of its points (see [6]). We refer to [2] and [6] for additional

Received by the editors November 5, 2002 and, in revised form, February 2, 2004.

2000 Mathematics Subject Classification. Primary 26B05; Secondary 46 G05.

Key words and phrases. Gradient range, derivative, bump, Morse-Sard theorem. 
references and background. The main result of this paper establishes the regular closedness of the gradient range for a class of bumps on the plane that is significantly larger than those satisfying (ii) above. We proceed with precise statements of the results.

Theorem. Let $b: \mathbb{R}^{2} \rightarrow \mathbb{R}$ be a $\mathcal{C}^{1}$-smooth bump such that $\nabla b$ has a modulus of continuity $\omega=\omega(t)$ satisfying $\omega(t) / \sqrt{t} \rightarrow 0$ as $t \searrow 0$. Then the gradient range $\nabla b\left(\mathbb{R}^{2}\right)$ is regularly closed, i.e., equals the closure of its interior.

The theorem is easily deduced from the following proposition that concerns bumps on $\mathbb{R}^{n}$. In its statement we denote by $\operatorname{dim} M$ the topological dimension of the set $M$.

Proposition 1. Let $n \geq 2$ and $b: \mathbb{R}^{n} \rightarrow \mathbb{R}$ be a $\mathcal{C}^{1}$-smooth bump such that $\nabla b$ has a modulus of continuity $\omega=\omega(t)$ satisfying $\omega(t) / \sqrt{t} \rightarrow 0$ as $t \searrow 0$.

If $G$ is an open set in $\mathbb{R}^{n}$ such that the intersection $G \cap \nabla b\left(\mathbb{R}^{n}\right)$ is non-empty, then $\operatorname{dim}\left(\nabla b\left(\mathbb{R}^{n}\right) \cap G\right) \geq 2$.

The theorem follows from the proposition, because a subset $M$ of $\mathbb{R}^{2}$ has topological dimension two precisely when it contains a non-empty open subset (see Th. IV 3 of 4 ).

The proof of Proposition 10 relies on the following Morse-Sard type result, in which the smoothness assumption does not depend on the dimension $n$ of the space. A similar result that does not involve Minkowski content and Hölder continuity can be found in $\left[\underline{6}\right.$. By $\mathcal{U}_{\varepsilon}(C)=\{y: \operatorname{dist}(y, C)<\varepsilon\}$ we denote the $\varepsilon$-neighbourhood of $C$. For $s \in[0, n]$, the $s$-dimensional lower Minkowski content of a set $C \subseteq \mathbb{R}^{n}$ is defined by

$$
\mathcal{M}_{*}^{s}(C)=\liminf _{\varepsilon \searrow 0}(2 \varepsilon)^{s-n} \mathcal{L}^{n}\left(\mathcal{U}_{\varepsilon}(C)\right)
$$

where $\mathcal{L}^{n}$ denotes the Lebesgue measure on $\mathbb{R}^{n}$.

A set $C$ in $\mathbb{R}^{n}$ is called an irreducible separator if there are two points $x$ and $y$ in $\mathbb{R}^{n}$ such that $C$ separates $x$ and $y$, but no proper subset $R$ of $C$ separates them.

Proposition 2. Assume $s \in(0,1), f \in \mathcal{C}^{1}\left(\mathbb{R}^{n}\right)$ and $\nabla f=0$ on a bounded irreducible separator $C$. If $\nabla f$ is $s$-Hölder and $\mathcal{M}_{*}^{n-1+s}(C)=0$, i.e.,

$$
\liminf _{\varepsilon \searrow 0} \frac{\mathcal{L}^{n}\left(\mathcal{U}_{\varepsilon}(C)\right)}{\varepsilon^{1-s}}=0,
$$

then $f$ is constant on $C$. The same is true if $\nabla f$ admits a modulus of continuity $\omega$ with $\lim _{\varepsilon \backslash 0} \omega(\varepsilon) / \varepsilon^{s}=0$ and $\mathcal{M}_{*}^{n-1+s}(C)<\infty$.

The organization of the paper is as follows. In Section 2 we set the notation, and state as lemmata a collection of elementary auxiliary results. The proofs of Propositions 1 and 2 are presented in Section 3.

\section{Preliminaries}

We use standard notation. In particular, $\|\cdot\|$ always denotes the euclidean norm on $\mathbb{R}^{n}, \mathrm{~B}(x, r)$ denotes the open ball with center $x$ and radius $r$, and $\mathrm{B}[x, r]$ the corresponding closed ball.

A non-decreasing, continuous function $\omega:[0, \infty) \rightarrow[0, \infty)$ with $\omega(0)=0$ is called a modulus of continuity of the vector field $V: \mathbb{R}^{n} \rightarrow \mathbb{R}^{n}$ if

$$
\|V(x)-V(y)\| \leq \omega(\|x-y\|)
$$


for all $x, y \in \mathbb{R}^{n}$. In particular, a vector field $V$ is $s$-Hölder if it admits a modulus of continuity of the form $\omega(t)=c t^{s}$ where $c$ is a constant.

We proceed with some auxiliary results of an elementary nature. The Lemmata 1 2, 4, 5 and 6 are similar to results contained in [6. We state them here for the convenience of the reader.

Lemma 1. Let $G \subseteq \mathbb{R}^{n}$ be bounded and open. Assume $f: \bar{G} \rightarrow \mathbb{R}$ is continuous, differentiable on $G, f=0$ on $\partial G$ and $f$ is not identically zero on $\bar{G}$. Then $0 \in \mathbb{R}^{n}$ is an interior point of $\nabla f(G)$.

Proof. Let $m=\max f(\bar{G})>0$. (In the opposite case $f$ should be replaced by $-f$.) Let $y \in \mathrm{B}(0, m / \operatorname{diam} G)$ and put $g(x)=f(x)-y \cdot x, x \in \bar{G}$. By continuity and compactness we can find $x_{0} \in \bar{G}$, such that $g\left(x_{0}\right)=\max g(\bar{G})$. It is not hard to see from the assumptions that $x_{0} \in G$, so $\nabla g\left(x_{0}\right)=0$, or $\nabla f\left(x_{0}\right)=y$. Hence $\mathrm{B}(0, m / \operatorname{diam} G) \subseteq \nabla f(G)$, and $0 \in \mathbb{R}^{n}$ is an interior point.

Lemma 2. Let $b: \mathbb{R}^{n} \rightarrow \mathbb{R}$ be a $\mathcal{C}^{1}$-smooth bump. The gradient range $\nabla b\left(\mathbb{R}^{n}\right)$ is a locally connected continuum that contains $0 \in \mathbb{R}^{n}$ in its interior.

Proof. The support of $b$ is contained in an open ball B, so $0 \in \operatorname{int} \nabla b(\mathrm{~B})=$ int $\nabla b\left(\mathbb{R}^{n}\right)$ by Lemma 1 The closure $\overline{\mathrm{B}}$ of the ball is a locally connected continuum, hence so is $\nabla b(\overline{\mathrm{B}})=\nabla b\left(\mathbb{R}^{n}\right)$ (see $\S 50 . \mathrm{II}$, Th. 5 of [7]).

A topological space $\mathcal{X}$ has (topological) dimension 0 at the point $x \in \mathcal{X}$, briefly $\operatorname{dim}_{x} \mathcal{X}=0$, if $x$ has arbitrarily small open neighborhoods with empty boundaries. In other words, given any open neighborhood $U$ of $x$ there exists an open neighborhood $V \subset U$, such that $\partial V=\emptyset$.

The space $\mathcal{X}$ is 0 -dimensional, briefly $\operatorname{dim} \mathcal{X}=0$, provided $\operatorname{dim}_{x} \mathcal{X}=0$ for all $x \in \mathcal{X}$. It is convenient to define the dimension of the empty set $\emptyset$ to be -1 .

Higher topological dimension is then defined by induction: The space $\mathcal{X}$ has dimension $n \in \mathbb{N}$ at $x$, briefly $\operatorname{dim}_{x} \mathcal{X}=n$, if it is false that $\operatorname{dim}_{x} \mathcal{X} \leq n-1$, but $x$ has arbitrarily small open neighborhoods with boundaries of dimension at most $n-1$. The space $\mathcal{X}$ is $n$-dimensional if $\max _{x \in \mathcal{X}} \operatorname{dim}_{x} \mathcal{X}=n$.

We shall apply the above definition only to subsets of $\mathbb{R}^{n}$ (considered with the topology inherited from $\mathbb{R}^{n}$ ).

Our reference on topological dimension is 4 to which we refer for a thorough discussion.

Let $\mathcal{X}$ be a topological space and $A, B$ and $C$ subsets. We say that $C$ separates $A$ and $B$ (in $\mathcal{X}$ ) if it is possible to split $\mathcal{X} \backslash C$ into two disjoint relatively open sets containing $A$ and $B$, respectively. That is, if there exist open sets $U$ and $V$ in $\mathcal{X}$, such that if we let $U^{\prime}=U \backslash C, V^{\prime}=V \backslash C$, then $\mathcal{X} \backslash C=U^{\prime} \cup V^{\prime}, A \subseteq U^{\prime}, B \subseteq V^{\prime}$ and $U^{\prime} \cap V^{\prime}=\emptyset$. (When $A=\{a\}, B=\{b\}$ and $C$ separates $A$ and $B$ we also say that $C$ separates $a$ and $b$, and that $C$ is a separator between $a$ and $b$.)

We say that $A$ and $B$ are irreducibly separated in $\mathcal{X}$ by $C$ (and that $C$ is an irreducible separator between $A$ and $B$ ) if $C$ separates $A$ and $B$ in $\mathcal{X}$, and any proper subset $R \subset C$ does not. We shall use this concept only when $A$ and $B$ are singletons. In this case it is not hard to show that an irreducible separator is always a closed set (see $\S 46$.VII, Th. 3 of [7]).

Lemma 3. Let $\mathcal{X}$ be a separable metric space, $A$ and $B$ closed and disjoint subsets of $\mathcal{X}$, and $M$ a subset of $\mathcal{X}$ of dimension at most $n \in \mathbb{N}$. Then there exists a closed set $C$ separating $A, B$ in $\mathcal{X}$, and with the property that $\operatorname{dim}(M \cap C) \leq n-1$. 
Proof. This is Prop. B, Ch. III, $\S 5$ of [4].

Lemma 4. Let $\mathcal{X}$ be a locally connected metric space.

(1) If $A$ is open and connected and $B$ is a component of $\mathcal{X} \backslash \bar{A}$, then the set $\partial B$ is an irreducible separator between each pair of points $a \in A$ and $b \in B$.

(2) Let $C$ be a closed set, $A$ and $B$ two distinct components of $\mathcal{X} \backslash C$, and let $a \in A$ and $b \in B$. The set $C$ is an irreducible separator between $a$ and $b$ if and only if $\partial A=C=\partial B$.

Proof. (1) is Th. 2 and (2) is Th. 1 in $\S 49$.VI of [7.

Lemma 5. Every irreducible separator between two distinct points of $\mathbb{R}^{n}$ is closed and connected.

Proof. This follows from $\S 57 . I I I .1$ of [7] that can be applied by $\S 57 . I .9(i)$ of [7].

Lemma 6. Let $f: \mathbb{R}^{n} \rightarrow \mathbb{R}$ be a $\mathcal{C}^{1}$ function, and $\Omega$ an open set in $\mathbb{R}^{n}$, such that $\nabla f=0$ on $\partial \Omega$ and such that $\partial \Omega$ is an irreducible separator between two points $x \in \Omega$ and $y \in \mathbb{R}^{n} \backslash \bar{\Omega}$. Then $f$ is constant on $\partial \Omega$ if the vector field

$$
V=\left\{\begin{array}{cl}
\nabla f & \text { in } \Omega \\
0 & \text { in } \mathbb{R}^{n} \backslash \Omega
\end{array}\right.
$$

is curl-free in the distributional sense on $\mathbb{R}^{n}$.

Proof. Suppose that $V$ is curl-free: $V_{, i}^{j}-V_{, j}^{i}=0$ holds in the distributional sense on $\mathbb{R}^{n}$ for each pair of indices $i, j=1, \ldots, n$. Using mollification and a standard result we find a $\mathcal{C}^{1}$ function $g: \mathbb{R}^{n} \rightarrow \mathbb{R}$ such that $V=\nabla g$. Now let $\Lambda_{1}$ and $\Lambda_{2}$ be the components of $\mathbb{R}^{n} \backslash \partial \Omega$ containing $x$ and $y$, respectively. From Lemma 4 (2) it follows that $\partial \Lambda_{1}=\partial \Omega=\partial \Lambda_{2}$. Obviously, $\Lambda_{1} \subseteq \Omega$ and $\Lambda_{2} \subseteq \mathbb{R}^{n} \backslash \bar{\Omega}$. Because $\Lambda_{1}$ is open and connected and $\nabla f=\nabla g$ there, we can assume that $g=f$ on $\bar{\Lambda}_{1}$. Next, $\nabla g=0$ on $\mathbb{R}^{n} \backslash \Omega$, so in particular, $\nabla g=0$ on $\Lambda_{2}$. Since $\Lambda_{2}$ is open and connected, $g$ must be constant on $\bar{\Lambda}_{2}=\Lambda_{2} \cup \partial \Omega$. But $f=g$ on $\bar{\Lambda}_{1}=\Lambda_{1} \cup \partial \Omega$, so $f$ is constant on $\partial \Omega$ too.

\section{THE PROOFS}

Proof of Proposition 1. Suppose the assertion of the proposition is false. Then we can clearly find a closed ball $\mathrm{B}_{0}=\mathrm{B}\left[y_{0}, R\right]$ centred at a point $y_{0}$ of $K=\nabla b\left(\mathbb{R}^{n}\right)$ such that $\operatorname{dim} M \leq 1$, where $M=K \cap \mathrm{B}_{0}$. In view of Lemma2 2 and since $n>1$ we must have $0 \notin \mathrm{B}\left[y_{0}, R\right]$. Let $0<r<s<R$, and consider the closed and disjoint sets $A=\mathrm{B}\left[y_{0}, r\right]$ and $B=\mathbb{R}^{n} \backslash \mathrm{B}\left(y_{0}, s\right)$. By virtue of Lemma B, we can find a closed set $C=C_{r, s}$ that separates $A$ and $B$ in $\mathbb{R}^{n}$ and such that $\operatorname{dim}(C \cap M) \leq 0$. Accordingly, there exist open sets $U=U_{r, s}, V=V_{r, s}$, such that $\mathbb{R}^{n} \backslash C=U \cup V$, $A \subseteq U, B \subseteq V$ and $U \cap V=\emptyset$. Since $\partial U \subseteq C$, we have found an open set $U_{r, s}$ in $\mathbb{R}^{n}$ such that $\mathrm{B}\left[y_{0}, r\right] \subseteq U_{r, s} \subseteq \mathrm{B}\left(y_{0}, s\right)$ and $\operatorname{dim}\left(M \cap \partial U_{r, s}\right)=0$. The latter implies that $M \cap \partial U_{r, s}$ is a totally disconnected set.

Select $x_{0} \in \mathbb{R}^{n}$ so that $\nabla b\left(x_{0}\right)=y_{0}$, and define for $0<r<s \leq R$, the sets $\Lambda_{r}, \Lambda_{r, s}$ and $\Lambda_{s}$ as the (connected) components of the open sets $(\nabla b)^{-1}\left(\mathrm{~B}\left(y_{0}, r\right)\right)$, $(\nabla b)^{-1}\left(U_{r, s}\right)$ and $(\nabla b)^{-1}\left(\mathrm{~B}\left(y_{0}, s\right)\right)$ that contain $x_{0}$, respectively. Then $\Lambda_{r}, \Lambda_{r, s}$ and $\Lambda_{s}$ are open connected sets, $\Lambda_{r} \subseteq \Lambda_{r, s} \subseteq \Lambda_{s}$ and $\nabla b\left(\partial \Lambda_{r}\right) \subseteq \partial \mathrm{B}\left(y_{0}, r\right)$, $\nabla b\left(\partial \Lambda_{r, s}\right) \subseteq M \cap \partial U_{r, s}$. Since $0 \notin M$ and $\nabla b \in M$ on $\Lambda_{s}$, it follows that $\Lambda_{s} \subseteq \operatorname{spt}(b)$, the support of $b$, and hence in particular that the sets are bounded. 
We assert that for each $r \in(0, R)$ and each sequence $\left\{r_{j}\right\}$ with $r_{j} \nearrow r$,

$$
\Lambda_{r}=\bigcup_{j=1}^{\infty} \Lambda_{r_{j}}
$$

and

$$
\Lambda_{r}=\bigcup_{j=1}^{\infty} \Lambda_{r_{j}, r}
$$

hold. To see this, let $x \in \Lambda_{r}$ and note that since $\Lambda_{r}$ is arcwise connected there is a curve $\gamma \subseteq \Lambda_{r}$ that connects $x$ and $x_{0}$. Now if we take $j$ so large that $r_{j}>$ $r-\operatorname{dist}\left(\nabla b(\gamma), \partial \mathrm{B}\left(y_{0}, r\right)\right) / 2$, then $\gamma$ does not intersect $(\nabla b)^{-1}\left(\partial \mathrm{B}\left(y_{0}, r_{j}\right)\right)$. Since $\Lambda_{r_{j}}$ is the component of $(\nabla b)^{-1}\left(\mathrm{~B}\left(y_{0}, r_{j}\right)\right)$ that contains $x_{0}$, it follows that $\gamma \subseteq \Lambda_{r_{j}}$, and thus in particular, $x \in \Lambda_{r_{j}}$, establishing (3.1). Then, (3.2) follows from the inclusions $\Lambda_{r} \subseteq \Lambda_{r, s} \subseteq \Lambda_{s}$.

Let $0<r<s<R$. Since $\Lambda_{r}$ and $\Lambda_{r, s}$ are bounded, the complements $\mathbb{R}^{n} \backslash \overline{\Lambda_{r}}$ and $\mathbb{R}^{n} \backslash \overline{\Lambda_{r, s}}$ both have precisely one unbounded component. Let $A_{r}$ and $A_{r, s}$ denote these, respectively. Next, let $\Omega_{r}$ and $\Omega_{r, s}$ denote the components of $\mathbb{R}^{n} \backslash \overline{A_{r}}$ and $\mathbb{R}^{n} \backslash \overline{A_{r, s}}$ that contain $x_{0}$, respectively. It follows from Lemma 4 that $\partial \Omega_{r}$ and $\partial \Omega_{r, s}$ are irreducible separators (between certain pairs of points). By Lemma 5 they are therefore also connected. Furthermore, we record the following facts: $\Omega_{r} \subseteq \Omega_{r, s} \subseteq \Omega_{s} \subseteq \operatorname{cospt}(b)$, the convex hull of the support of $b, \Lambda_{r} \subseteq \Omega_{r}, \Lambda_{r, s} \subseteq$ $\Omega_{r, s}$ and $\partial \Omega_{r} \subseteq \partial \Lambda_{r}, \partial \Omega_{r, s} \subseteq \partial \Lambda_{r, s}$. (For example, $\partial \Omega_{r} \subseteq \partial \Lambda_{r}$ follows by applying twice the general result stating that for a closed subset $B$ of $\mathbb{R}^{n}$ the boundary of every component of $\mathbb{R}^{n} \backslash B$ is contained in $\partial B$.)

Now recall that $\nabla b \in M \cap \partial U_{r, s}$ on $\partial \Lambda_{r, s}$. Consequently, $\nabla b$ maps the connected space $\partial \Omega_{r, s}$ continuously into the totally disconnected space $M \cap \partial U_{r, s}$ and is therefore constant: $\nabla b=y_{r, s}$ on $\partial \Omega_{r, s}$. Next we deduce that $\nabla b$ is also constant on each of the boundaries $\partial \Omega_{s}, s \in(0, R)$, by observing that for each $x \in \partial \Omega_{s}$,

$$
\lim _{r / s} \operatorname{dist}\left(x, \partial \Omega_{r, s}\right)=0 .
$$

The routine verification of (3.3) is left to the interested reader.

Let us summarize what has been achieved so far. To each $r \in(0, R)$ there is assigned an open connected set $\Omega_{r}$ contained in $\operatorname{cospt}(b), \partial \Omega_{r}$ is an irreducible separator (between certain pairs of points), and $\nabla b=y_{r}$ on $\partial \Omega_{r}$, where $y_{r} \in$ $M \cap \partial \mathrm{B}\left(y_{0}, r\right)$. Furthermore, $\Omega_{r} \subseteq \Omega_{s}$ when $r<s$. Moreover, since $y_{r} \neq y_{s}$, $\partial \Omega_{r} \cap \partial \Omega_{s}=\emptyset$ and hence $\bar{\Omega}_{r} \subset \Omega_{s}$ when $r<s$.

Assume that for some $r \in(0, R)$ the function $x \mapsto b(x)-y_{r} \cdot x$ is constant, say equal to $c$, on the boundary $\partial \Omega_{r}$. Put $f(x)=b(x)-y_{r} \cdot x-c$ and $G=\Omega_{r}$. Since $x_{0} \in G$ and $\nabla f\left(x_{0}\right)=y_{0}-y_{r} \neq 0$, it follows that $f$ is not identically zero on $G$, and therefore, in view of Lemma 1, that $0 \in \mathbb{R}^{n}$ is an interior point of $\nabla f(G)$. But this implies that $y_{r}$ is an interior point of $\nabla b\left(\Omega_{r}\right)$, and hence in particular also an interior point of $\nabla b\left(\mathbb{R}^{n}\right)$, leading to the contradiction that $n=\operatorname{dim}_{y_{r}} \nabla b\left(\mathbb{R}^{n}\right)<2$.

The proof is therefore complete if we show that $x \mapsto b(x)-y_{r} \cdot x$ is constant on $\partial \Omega_{r}$ for some $r \in(0, R)$. We shall do this by use of Proposition 2 after we compute, for suitable $r$, the $(n-1 / 2)$-dimensional Minkowski content of $\partial \Omega_{r}$.

Define $h(r)=\mathcal{L}^{n}\left(\Omega_{r}\right), r \in(0, R)$. Then $h$ is a non-decreasing function and $h(r) \leq \mathcal{L}^{n}\left(\Omega_{R}\right)<\infty$ for $r \in(0, R)$. The function $h$ is therefore in particular 
differentiable almost everywhere; hence we may select $r_{0} \in(0, R)$ and an $\varepsilon_{0}>0$ so small that with $m=2 h^{\prime}\left(r_{0}\right)+1<\infty$ we have

$$
h\left(r_{0}+\varepsilon\right)-h\left(r_{0}-\varepsilon\right)<m \varepsilon \quad \text { for } \varepsilon \in\left(0, \varepsilon_{0}\right) .
$$

Fix $\varepsilon>0$ with $\omega(\varepsilon)<r_{0} / 2$. Let $x \in \mathcal{U}_{\varepsilon}\left(\partial \Omega_{r_{0}}\right)$, i.e. there exists $x^{\prime} \in \partial \Omega_{r_{0}}$ so that $\left\|x-x^{\prime}\right\|<\varepsilon$. Since $\nabla b\left(x^{\prime}\right)=y_{r_{0}} \in \partial \mathrm{B}\left(y_{0}, r_{0}\right)$ and $\left\|\nabla b\left(x^{\prime}\right)-\nabla b\left(x^{\prime \prime}\right)\right\| \leq \omega(\varepsilon)$ for all $x^{\prime \prime} \in \mathrm{B}\left(x^{\prime}, \varepsilon\right)$, we must have

$$
\nabla b\left(\mathrm{~B}\left(x^{\prime}, \varepsilon\right)\right) \subset \mathrm{B}\left(y_{r_{0}}, 2 \omega(\varepsilon)\right) \subset \mathrm{B}\left(y_{0}, r_{0}+2 \omega(\varepsilon)\right) \backslash \mathrm{B}\left[y_{0}, r_{0}-2 \omega(\varepsilon)\right] .
$$

Hence $\mathrm{B}\left(x^{\prime}, \varepsilon\right)$ does not intersect the boundaries $\partial \Omega_{r_{0}+2 \omega(\varepsilon)}, \partial \Omega_{r_{0}-2 \omega(\varepsilon)}$, and because $x^{\prime} \in \partial \Omega_{r_{0}} \subset \Omega_{r_{0}+2 \omega(\varepsilon)} \backslash \overline{\Omega_{r_{0}-2 \omega(\varepsilon)}}$ and $\mathrm{B}\left(x^{\prime}, \varepsilon\right)$ is connected, it follows that $\mathrm{B}\left(x^{\prime}, \varepsilon\right) \subseteq \Omega_{r_{0}+2 \omega(\varepsilon)} \backslash \overline{\Omega_{r_{0}-2 \omega(\varepsilon)}}$. Consequently,

$$
\mathcal{U}_{\varepsilon}\left(\partial \Omega_{r_{0}}\right) \subseteq \Omega_{r_{0}+2 \omega(\varepsilon)} \backslash \overline{\Omega_{r_{0}-2 \omega(\varepsilon)}}
$$

when $\varepsilon>0$ and $\omega(\varepsilon)<r_{0} / 2$. Now for $\varepsilon>0$ so small that $\omega(\varepsilon)<\min \left\{r_{0}, \varepsilon_{0}\right\} / 2$ we get in view of (3.4) that $\mathcal{L}^{n}\left(\mathcal{U}_{\varepsilon}\left(\partial \Omega_{r_{0}}\right)\right) \leq 2 m \omega(\varepsilon)$, and therefore

$$
\mathcal{M}_{*}^{n-\frac{1}{2}}\left(\partial \Omega_{r_{0}}\right) \leq \liminf _{\varepsilon \backslash 0} \frac{2 m \omega(\varepsilon)}{\sqrt{2 \varepsilon}}=0 .
$$

Finally, we may use Proposition 2 with $s=1 / 2$ to conclude that $g(x)=b(x)-y_{r_{0}} \cdot x$ is constant on the irreducible separator $\partial \Omega_{r_{0}}$.

Proof of Proposition 2, By the assumption, $C$ is an irreducible separator between two points $x$ and $y$. Let $\Omega$ and $\Lambda$ be the components of $\mathbb{R}^{n} \backslash C$ containing the points $x$ and $y$, respectively. At least one of them is bounded and we may assume that it is $\Omega$. By Lemma $4(2), \partial \Omega=C=\partial \Lambda$.

Let $d(x)=\operatorname{dist}(x, \partial \Omega)=\inf \{\|x-y\|: y \in \partial \Omega\}$ denote the distance from $x$ to the boundary $\partial \Omega$. There exists a function $\delta: \Omega \rightarrow \mathbb{R}$ that is $\mathcal{C}^{\infty}$ on $\Omega$ and has the properties $d(x) / c_{0} \leq \delta(x) \leq c_{0} d(x),\|\nabla \delta(x)\| \leq 1$ and $\left|\partial^{\alpha} \delta(x)\right| \leq c_{|\alpha|} d(x)^{1-|\alpha|}$ for all $x \in \Omega$ and any multi-index $\alpha$, where $c_{|\alpha|} \in[1, \infty)$ are constants. We refer to [8], Th. 2, p. 171, for an explicit construction of such a regularized distance $\delta$.

For each $t>0$, define the auxiliary set $\Omega^{(t)}=\{x \in \Omega: \delta(x)>t\}$. In view of the (classical) Morse-Sard theorem, $\mathcal{L}^{1}(\delta(\{x \in \Omega: \nabla \delta(x)=0\}))=0$, so $\nabla \delta \neq 0$ on $\partial \Omega^{(t)}$ for almost all $t$. It follows that $\Omega^{(t)}$ has a $\mathcal{C}^{1}$ boundary with exterior unit normal $N=-\nabla \delta /\|\nabla \delta\|$ for almost all $t$.

Obviously, $\partial \Omega^{(t)} \subseteq \mathcal{U}_{2 c_{0} t}(\partial \Omega)$. By use of the coarea formula we estimate

$$
\mathcal{L}^{n}\left(\mathcal{U}_{\varepsilon}(\partial \Omega)\right) \geq \frac{1}{n c_{1}} \int_{0}^{\frac{\varepsilon}{2 c_{0}}} \mathcal{H}^{n-1}\left(\partial \Omega^{(t)}\right) d t
$$

whereby it follows that we can select $t=t(\varepsilon) \in\left(0, \frac{\varepsilon}{2 c_{0}}\right)$, such that $\Omega^{(t)}$ has a $\mathcal{C}^{1}$ boundary and

$$
\mathcal{H}^{n-1}\left(\partial \Omega^{(t)}\right) \leq \frac{2 n c_{0} c_{1} \mathcal{L}^{n}\left(\mathcal{U}_{\varepsilon}(\partial \Omega)\right)}{\varepsilon} .
$$

We now consider the vector field

$$
V=\left\{\begin{array}{cc}
\nabla f & \text { in } \Omega, \\
0 & \text { else. }
\end{array}\right.
$$


Let $\varphi \in \mathcal{C}_{c}^{\infty}\left(\mathbb{R}^{n}\right)$ denote a smooth and compactly supported test function and compute for distinct $i, j$ from $\{1, \ldots, n\}$ :

$$
\left\langle V_{, j}^{i}-V_{, i}^{j}, \varphi\right\rangle=\int_{\Omega}\left(f_{, j} \varphi_{, i}-f_{, i} \varphi_{, j}\right) .
$$

By the dominated convergence theorem, $\int_{\Omega^{(t)}}\left(f_{, j} \varphi_{, i}-f_{, i} \varphi_{, j}\right) \rightarrow \int_{\Omega}\left(f_{, j} \varphi_{, i}-f_{, i} \varphi_{, j}\right)$ as $t \searrow 0$. For those $t$ for which $\Omega^{(t)}$ has a $\mathcal{C}^{1}$ boundary we get, using a standard mollifier $\left\{\rho_{s}\right\}$ and integration by parts,

$$
\begin{aligned}
\int_{\Omega^{(t)}}\left(f_{, i} \varphi, j-f, j \varphi_{, i}\right) & =\lim _{s \searrow 0} \int_{\Omega^{(t)}} \operatorname{div}\left(\varphi\left(\rho_{s} \star f_{, i}\right) e_{j}-\varphi\left(\rho_{s} \star f_{, j}\right) e_{i}\right) \\
& =\lim _{s \searrow 0} \int_{\partial \Omega^{(t)}}\left(\varphi\left(\rho_{s} \star f_{, i}\right) N^{j}-\varphi\left(\rho_{s} \star f_{, j}\right) N^{i}\right) \\
& =\int_{\partial \Omega^{(t)}} \varphi\left(f_{, i} N^{j}-f_{, j} N^{i}\right),
\end{aligned}
$$

where $N=\left(N^{1}, \ldots, N^{n}\right)$ denotes the exterior unit normal to $\partial \Omega^{(t)}$. This is in particular true if we take $t=t(\varepsilon)$. For this choice of $t$ we estimate the last term using (3.5) (denoting $c=2 n c_{0} c_{1}$ ):

$$
\left|\int_{\Omega^{(t)}}\left(f_{, j} \varphi_{, i}-f_{, i} \varphi, j\right)\right| \leq \max |\varphi| \omega(2 t) \mathcal{H}^{n-1}\left(\partial \Omega^{(t)}\right) \leq \max |\varphi| \frac{c \omega(\varepsilon) \mathcal{L}^{n}\left(\mathcal{U}_{\varepsilon}(\partial \Omega)\right)}{\varepsilon},
$$

where $\omega$ denotes the (nondecreasing) modulus of continuity of $\nabla f$. By the assumption (either one of the two versions), the right-hand side $R(\varepsilon)$ has $\lim \inf _{\varepsilon \backslash 0} R(\varepsilon)=$ 0 , so we have shown that $\left\langle V_{, j}^{i}-V_{, i}^{j}, \varphi\right\rangle=0$. It follows that $V$ is curl-free on $\mathbb{R}^{n}$, and hence by virtue of Lemma 6 , that $f$ is constant on the irreducible separator $\partial \Omega$. The proof is finished.

\section{ACKNOWLEDGEMENT}

The first author was supported by grants GAČR 201/02/D111, MSM 113200007 and GAČR 201/03/0931. The second author was partially supported by EPSRC grant GR/R25002/01. We are grateful to the referee for many useful comments and for pointing out the reference [5].

\section{REFERENCES}

[1] D. Azagra \& M. Jiménez-Sevilla. On the size of the sets of gradients of bump mappings and starlike bodies on the Hilbert space. Bull. Soc. Math. France 130 (2002), no. 3, 337-347. MR:1943881 (2003k:46056)

[2] M. Fabian, O. Kalenda \& J. Kolár. Filling analytic sets by the derivatives of $C^{1}$-smooth bumps. Proc. Amer. Math. Soc., to appear.

[3] T. GASPARI. On the range of the derivative of a real-valued function with bounded support. Studia Math. 153 (2002), no. 1, 81-99. MR1948929 (2003k:46057)

[4] W. Hurewicz \& H. Wallman. Dimension theory. Princeton University Press, 1948. MR0006493 (3:312b)

[5] P. Hartman \& L. Nirenberg. On spherical image maps whose Jacobians do not change sign. Amer. J. Math. 81 (1959), 901-920. MR0126812(23:A4106)

[6] J. Kolář \& J. Kristensen. The set of gradients of a bump. Max-Planck-Institute MiS, Leipzig, Preprint Nr. 64/2002. 
[7] C. Kuratowski. Topology Vol. II. English Edition. Academic Press and PWN-Polish Scientific Publishers, 1966. MR0259835 (41:4467)

[8] E.M. Stein. Singular integrals and differentiability properties of functions. Princeton University Press, 1970. MR0290095 (44:7280)

Department of Mathematical Analysis, Faculty of Mathematics and Physics, Charles University, Sokolovská 83, 18675 Praha 8, Czech Republic

E-mail address: kolar@karlin.mff.cuni.cz

Mathematical Institute, 24-29 St Giles', University of Oxford, Oxford OX1 3LB, United Kingdom

E-mail address: kristens@maths.ox.ac.uk 